OPEN ACCESS

Edited by:

Xiaofei $\mathrm{Hu}$,

Army Medical University, China

Reviewed by:

Ivan V. Brak

State Scientific Research Institute of

Physiology and Basic

Medicine, Russia

Karsten Wrede,

University of

Duisburg-Essen, Germany

*Correspondence:

Zihao Zhang

zhzhang@ibp.ac.cn

Specialty section:

This article was submitted to Applied Neuroimaging,

a section of the journal

Frontiers in Neurology

Received: 26 April 2021

Accepted: 08 July 2021

Published: 16 August 2021

Citation:

Wei N, Zhang $X$, An J, Zhuo $Y$ and Zhang Z (2021) A Processing Pipeline for Quantifying Lenticulostriate Artery Vascular Volume in Subcortical Nuclei.

Front. Neurol. 12:700476.

doi: 10.3389/fneur.2021.700476

\section{A Processing Pipeline for Quantifying Lenticulostriate Artery Vascular Volume in Subcortical Nuclei}

\author{
Ning Wei ${ }^{1}$, Xianchang Zhang ${ }^{2}$, Jing An ${ }^{3}$, Yan Zhuo ${ }^{4,5}$ and Zihao Zhang ${ }^{4,5 *}$ \\ ${ }^{1}$ China National Clinical Research Center for Neurological Diseases, Beijing Tiantan Hospital, Capital Medical University, \\ Beijing, China, ${ }^{2}$ MR Collaboration, Siemens Healthcare Ltd., Beijing, China, ${ }^{3}$ Siemens Shenzhen Magnetic Resonance Ltd., \\ Shenzhen, China, ${ }^{4}$ State Key Laboratory of Brain and Cognitive Science, Institute of Biophysics, Chinese Academy of \\ Sciences, Beijing, China, ${ }^{5}$ University of Chinese Academy of Sciences, Beijing, China
}

Lenticulostriate arteries (LSAs) supply blood to the basal ganglia region. Its lesion causes lacunar stroke and resulting neurological syndromes. However, due to its small caliber and large individual variance, the evaluation of LSAs was limited to descriptive and objective measurements. In this study, we aimed to develop a post-processing method to quantify LSAs in subcortical regions and compare their vascular volume to conventional LSA measurements. A processing pipeline was designed to extract subcortical areas in individual spaces while screening out vessels. The vascular volume of LSAs in the subcortical region was calculated from time-of-flight-magnetic resonance angiography (TOF-MRA) at 7 Tesla. The reproducibility was tested to be good for the vascular volume $\left(n=5, I C C_{A}=0.84\right)$. Comparing the results to conventional measurements, the vascular volume was significantly correlated with the number of branches $(r=0.402, p<0.001)$ and the length ( $r=0.246, p=0.032$ ) of LSAs. By applying the method to a group of healthy volunteers $(n=40)$, we found that most LSAs crossing through the putamen which thereby has the highest vascular density among subcortical nuclei. In general, we proposed a semi-automated processing pipeline for quantifying the vascular volume of LSAs in subcortical regions. The novel method was tested to be robust and provided reasonable results. This method revealed spatial relationships among the perforating arteries and basal ganglia. The vascular volume can be used to evaluated blood supply of subcortical regions, benefiting the radiologic evaluation of neurodegenerative diseases caused by small vascular lesions.

Keywords: TOF-MRA, lenticulostriate artery, subcortical nuclei, $7 \mathrm{~T}$, vascular volume

\section{INTRODUCTION}

Lenticulostriate arteries (LSAs) represent the major microvasculature of the middle cerebral arteries and supply blood to the basal ganglia and internal capsules $(1,2)$. The occlusion of LSAs will cause lacunar infarcts in subcortical structures, leading to motor deficits, sensory deficits, and cognitive dysfunction (3). Imaging LSAs could be useful for clinical applications and provide insights into the mechanisms underlying cerebral microvascular disease development. Digital subtraction angiography (DSA) has been the gold standard for intracranial vascular imaging (4), but this method requires intravenous contrast and is not suitable for routine evaluations. With advances in imaging techniques, magnetic resonance angiography (MRA) has been widely used in clinical 
medicine because of its non-invasiveness. In the past decade, time-of-flight magnetic resonance angiography (TOF-MRA) at 7 Tesla (7T) has been shown to produce markedly superior blood-to-tissue contrast, which has enabled the non-invasive visualization of human brain microvasculature, in vivo, especially the microvasculature of $\operatorname{LSAs}(5,6)$. Lenticulostriate artery angiography is useful as a diagnostic tool in clinical and research settings for detecting diseases, such as small vessel disease, lacunar infarcts, and vascular dementia. Recent studies have found LSA abnormalities in patients with hypertension (7), lacunar infarcts (8), and vascular dementia (9).

The distribution and morphological characteristics of cerebral small vessels play important roles in diagnosis and treatment of various cerebrovascular diseases $(10,11)$. However, the analysis tools of LSAs, particularly the quantitative characterization, are still limited. In most studies, the number of LSA stems and branches arising from the first segment of the bilateral ACAs and MCAs are counted manually $(7,9,12)$. Some studies have measured the angles, lengths, and curvatures of the longest LSAs on $2 \mathrm{D}$ maximum intensity projection (MIP) images $(6,13,14)$ or $3 \mathrm{D}$ extracted vessels $(15,16)$. In addition, LSA orifices (8) and vascular densities obtained with rough threshold segmentations (17) have also been proposed. However, due to morphologic variations in LSA structures, these quantification methods have poor sensitivity and stability in being able to evaluate patient groups and sometimes have led to controversial clinical study results $(12,18)$.

Based on LSA spatial relationships and those of its supplying territories, our study introduces a novel method to quantify LSA vascular volume in subcortical areas to estimate basal ganglia and internal capsule blood supplies. A semi-automatic post-processing pipeline was designed and adjusted to produce LSA vascular volume, objectively. Conventional quantitative indicators, such as the number of branches and lengths of the LSAs were compared with LSA vascular volume.

\section{MATERIALS AND METHODS}

\section{Participants}

A total of 45 participants were enrolled in this study, separated into validation group $\left(\mathrm{G}_{\mathrm{val}}\right)$ and application group $\left(\mathrm{G}_{\mathrm{app}}\right) . \mathrm{G}_{\mathrm{val}}$ was used to validate the method and assess its robustness and reproducibility. It included five healthy participants (Aged $25 \pm 2.3$ years, 3 males). These participants underwent MRI acquisition twice on the same day, with repositioning between two scans. $G_{\text {app }}$ was applied to find the prevalence of LSAs in basal ganglia region and formed by 40 healthy volunteers (Aged $40.7 \pm 9.7$ years, 21 males). Informed consent was obtained from all participants and was approved by the local institutional review board.

\section{Magnetic Resonance Imaging}

All MRI scans were performed on a 7T whole-body research MR scanner (Siemens Healthcare, Erlangen, Germany). A birdcage transmission and a 32-channel receiving head coil (Nova Medical, MA, USA) were used for all scanning procedures.
T1-weighted magnetization-prepared rapid gradient echo (T1w MPRAGE) and TOF-MRA were collected for every participant.

Two protocols of TOF-MRA sequence were optimized to visualize LSAs. To evaluate the reproducibility of our method, whole-brain TOF-MRA with isotropic $0.40 \mathrm{~mm}$ resolution was acquired in $G_{v a l}$ with the following parameters: field of view $($ FOV $)=205 \times 175 \times 107 \mathrm{~mm}^{3}$, number of slabs $=6$, repetition time $(\mathrm{TR})=18 \mathrm{~ms}$, echo time $(\mathrm{TE})=4.03 \mathrm{~ms}$, flip angle $(\mathrm{FA})=22^{\circ}$, bandwidth $(\mathrm{BW})=158 \mathrm{~Hz} / \mathrm{Px}$, generalized auto-calibrating partial parallel acquisition (GRAPPA) factor = 3 , time of acquisition (TA) $=9 \min 28 \mathrm{~s}$. For the $\mathrm{G}_{\mathrm{app}}$, single-slab TOF-MRA was optimized to shorten the acquisition time (9). The imaging slab was positioned obliquely axial covering the bottom of the MCA and basal ganglia. Detailed parameters were FOV = $180 \times 135 \times 52 \mathrm{~mm}^{3}$, resolution $=0.23 \times 0.23 \times 0.36 \mathrm{~mm}^{3}$, TR $=15 \mathrm{~ms}, \mathrm{TE}=3.57 \mathrm{~ms}, \mathrm{FA}=20^{\circ}, \mathrm{BW}=151 \mathrm{~Hz} / \mathrm{Px}, \mathrm{GRAPPA}$ factor $=2, \mathrm{TA}=8 \mathrm{~min} 20 \mathrm{~s}$. The T1w MPRAGE was obtained for structural images in both groups, with the following parameters: FOV $=224 \times 224 \times 179 \mathrm{~mm}^{3}$, resolution $=0.70 \times 0.70 \times 0.70$ $\mathrm{mm}^{3}, \mathrm{TR}=3,000 \mathrm{~ms}, \mathrm{TE}=3.23 \mathrm{~ms}$, inversion time $=1,050 \mathrm{~ms}$, $\mathrm{FA}=8^{\circ}, \mathrm{BW}=320 \mathrm{~Hz} / \mathrm{Px}, \mathrm{GRAPPA}$ factor $=3, \mathrm{TA}=5 \min 54 \mathrm{~s}$.

\section{Image Analysis \\ Pre-processing}

The pre-processing was performed in FSL software, a comprehensive library of analytic tools (19). The processing workflow is shown in Figure 1. First, the T1w anatomical dataset was registered to the Montreal Neurological Institute (MNI) brain atlas. Non-linear registrations (FNIRT) were used following affine linear transformations (FLIRT) for maximal accuracy. Second, we chose the regions of interest (ROI) indicating the LSA supply territories to build a mask in the MNI space. The selected areas included the putamen, globus pallidus, and caudate nuclei from the Harvard-Oxford Subcortical Structural Atlas and the posterior limbs of the internal capsule from the JHU ICBM-DTI-81 White-Matter labels. On the other hand, the TOF-MRA dataset was aligned to its corresponding $\mathrm{T} 1 \mathrm{w}$ anatomical dataset. The $\mathrm{N} 4$ bias correction was applied to resolve signal inhomogeneity caused by the dielectric effects of ultra-high fields (20). Finally, rigid and non-linear transformations were concatenated to transform the ROI masks into individual TOF-MRA spaces.

\section{Vessel Enhancement and Filtering}

To enhance vessel-to-tissue contrast and suppress non-vascular structures and image noise, we used vessel enhancement filters from the Vascular Modeling Toolkit (21). One voxel of LSA was selected as a seed point. Based on the morphology of LSAs and spatial resolutions of TOF-MRA, vessel enhancement filter parameters were optimized as: minimum vessel diameter $=1$ voxel, maximum vessel diameter $=5$ voxels, vessel contrast $=100$, suppress plates $=10 \%$, and suppress blobs $=40 \%$. Then, we used the previously generated ROI masks to extract enhanced vasculatures to calculate the volume of LSAs, as shown in Figure 1.

Blood vessel volume was analyzed with the MATLAB 2016 software program. We applied a volume ratio scheme to find 


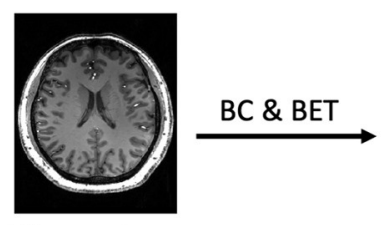

T1w anatomy

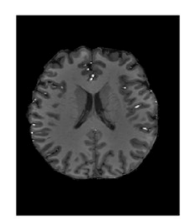

T1w anatomy
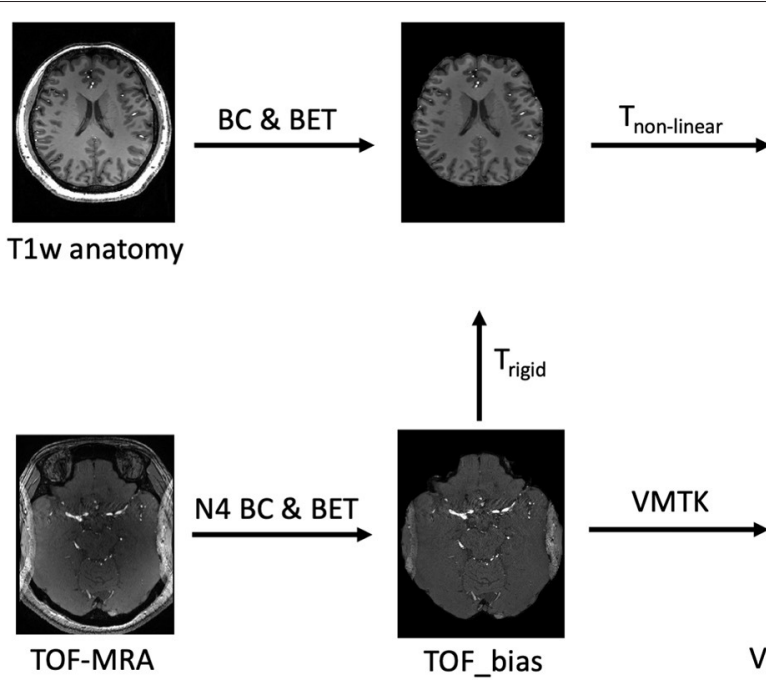

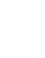

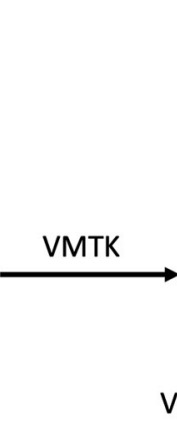

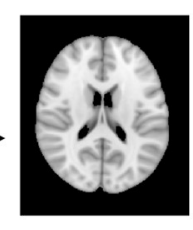

MNI

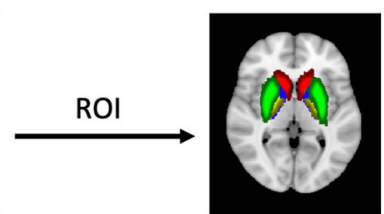

ROI in MNI space

FIGURE 1 | The workflow for calculating lenticulostriate artery (LSA) vascular volume. BC, bias correction; BET, brain extraction tool using FSL software; VMTK, Vascular Modeling Toolkit.

an appropriate threshold for screening out complete vessels while filtering out noise from the parenchymal tissues. The thresholds were adjusted until vessel-to-tissue volume ratios changed $>0.2 \%$. The vascular volume was defined as the number of voxels above the threshold multiplied by the imaging resolution.

\section{Quantifying the Morphologic Characteristics of Lenticulostriate Arteries}

Three dimensional (3D) MRA image reconstructions and analyses were performed using a free and open-source code software from Horos (https://horosproject.org). We counted the number of LSA stems and branches arising from the first segment of the bilateral ACAs (A1) and MCAs (M1). Only blood vessels of the LSA-supplying territories (the previously mentioned ROIs) were counted. Stems were defined as LSAs directly connected to A1s or M1s. Branches were defined as daughter vessels arising from parent LSA stems without any subordinate branches. If a trunk had no branches, it was recorded as both stem and branch. To measure the maximal length of the LSAs, MIP was reconstructed in the coronal view with a slab thickness of $28 \mathrm{~mm}$ covering the LSAs and MCAs. The maximal length was determined as the distance from MCA to the visible end of the longest LSA.

\section{Statistical Analyses}

The inter-class correlation coefficient (ICC) was used as the measure for reproducibility, assessing the absolute agreement $\left(\mathrm{ICC}_{\mathrm{A}}\right)$ between both scans of $\mathrm{G}_{\mathrm{val}}$. In addition, the data of $\mathrm{G}_{\mathrm{app}}$ was used for quantitative analysis. All the quantitative data were presented as means \pm standard deviations (SDs). The level of significance was set at $p<0.05$. The volume of LSAs calculated with our new method was compared with conventional LSA measurements using Pearson's correlation coefficient. All statistical analyses were carried out using SPSS vision 21 software.

\section{RESULTS}

Figure 2 shows an example of subcortical ROIs and LSA voxels. The subcortical nuclei and basal ganglia were well-segmented according to the anatomic images. The LSA vasculature was clearly demonstrated after vessel enhancement and filtering. The volumes of subcortical ROIs have an $\mathrm{ICC}_{\mathrm{A}}=0.98(95 \% \mathrm{CI}=$ 0.88-0.99), confirming excellent registration for ROI definition. The inter-scan reproducibility of quantifying LSA volume was good with an $\mathrm{ICC}_{\mathrm{A}}=0.84(95 \% \mathrm{CI}=0.47-0.96)$. An example of reproducibility test was exhibited in Figure 3. The discrepancies between the repeated scans occurred mostly for tiny branches of LSA which was sensitive to partial volume effect.

Two participants in $G_{\text {app }}$ were excluded from the analyses because voxels of large arteries were involved in the basilar ROI and contaminated the results. In total, 38 participants (76 hemispheres) were used for the statistical analyses. The LSA voxel numbers in the subcortical nuclei and internal capsules were $998.01 \pm 206.88$, which corresponded to a volume of $19.01 \pm 0.45 \mathrm{~mm}^{3}$. There were $5.71 \pm 1.41 \mathrm{LSA}$ branches and $3.39 \pm 1.07$ stems in each hemisphere, and the length of the longest branch was $3.44 \pm 0.53 \mathrm{~cm}$. The vascular volume was significantly correlated with the number of LSA branches $(r=$ $0.402, p<0.001)$ and the LSA lengths $(r=0.246, p=0.032)$, but no correlation was found between the vascular volume and the number of LSA stems $(r=0.062, p=0.596)$, as shown in Figure 4. The LSA voxel numbers in different subcortical regions (putamen, globus pallidus, caudate nucleus, and internal capsule) were further analyzed. We found that $90 \%$ of the visible perforating branches funneled through the putamen, which can be observed in Figure 2. 

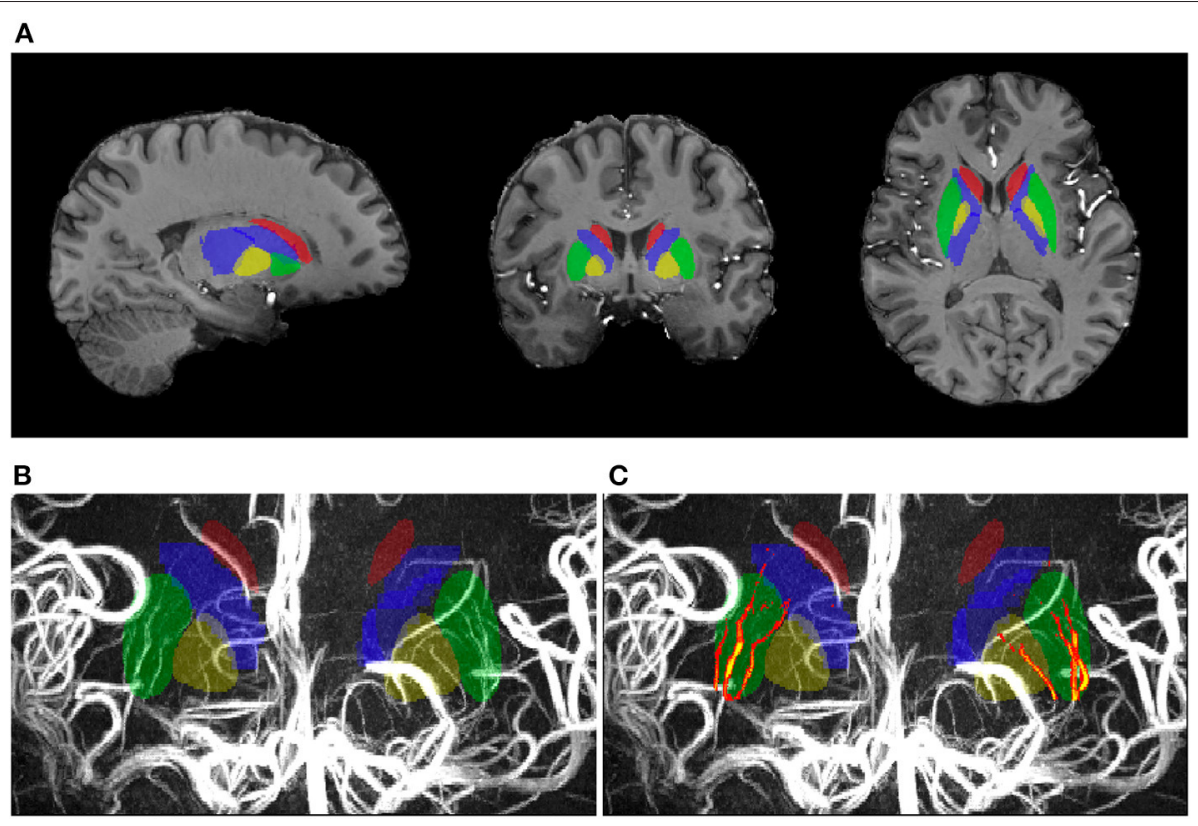

FIGURE 2 | The subcortical regions of interest (ROIs) and LSA vascular volume in one participant. (A) The ROls in the anatomical image; (B,C) The vascular voxels identified in the ROls concide well with the vasculature in the raw image.

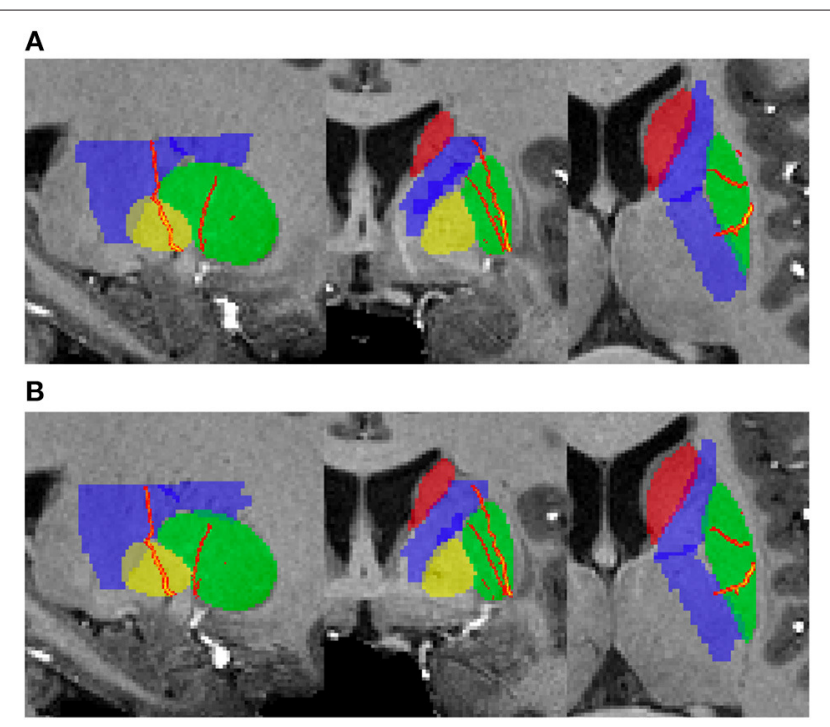

FIGURE 3 | The extracted LSA vasculatures of one hemisphere in the reproducibility testing. (A) The first scan; (B) The second scan.

\section{DISCUSSION}

Our study proposed a novel method for quantifying perforating arteries in subcortical regions. LSA vascular volume, equivalent to voxel numbers, reflected regions of subcortical blood supply. In previous studies, stems, branches, and lengths were used to describe LSA morphologies. Compared with these traditional measurements, the vascular volume in subcortical
ROIs was significantly correlated with the number of LSA branches and the LSA lengths. Conversely, LSA vascular volume was not correlated with the number of LSA stems, which could be explained by larger perforating arteries dividing into numerous branches before entering the brain substance $(22,23)$. Lenticulostriate artery volume assessments were able to estimate blood supplies in the subcortical regions more appropriately.

This new analytic method has two main advantages over traditional methods. First, the vascular volume is comprehensive representations of LSA numbers, lengths, and diameters. In a previous study, negative correlations were reported between LSA numbers and diameters (the higher the number of branches, the smaller the branch diameters, and vice versa) (24). Vascular volume measurements provided more comprehensive LSA quantifications in the subcortical regions, which was likely related to the blood perfusion in this area. In addition, the analytic methods represent an automatic post-processing pipeline that avoids the subjective bias of traditional measurements. In previous studies, investigators had to manually count the number of branches and draw curves to obtain diameter lengths. These operations depended more or less on subjective vasculature definitions. However, our new method extracted LSAs in an automated way eliminating subjective influence. The reproducibility testing gave robust and consistent results, owing to its fixed and deterministic behavior. Therefore, this novel method would be suitable for perforating artery analyses in studies with larger populations.

According to previous studies, a consensus has been reached that LSA imaging has important clinical implications and provides insights into the mechanisms underlying the 


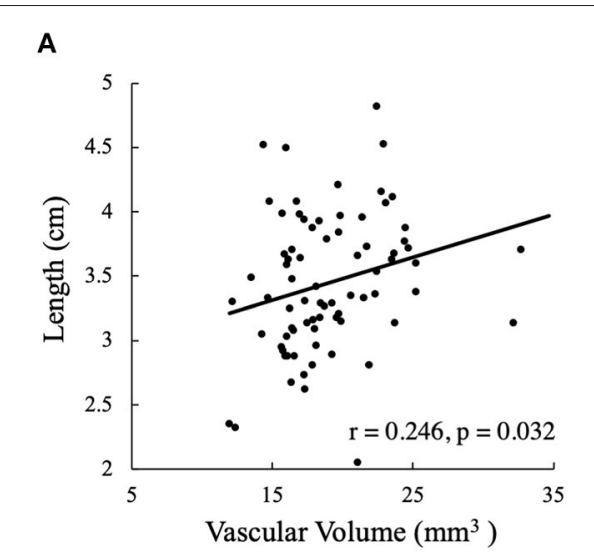

B

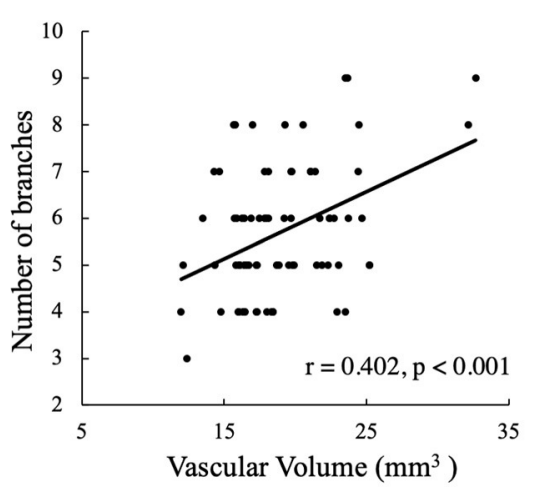

C

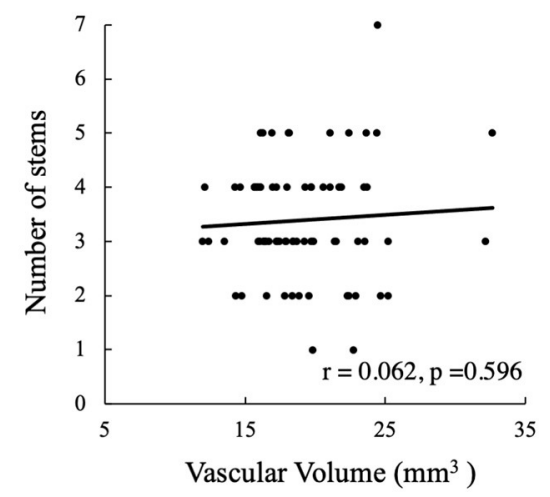

FIGURE 4 | Correlation analyses of lenticulostriate artery (LSA) volume and conventional measurements; LSA vascular volume vs. (A) LSA lengths, (B) the number of LSA branches, and (C) the number of LSA stems.

development of cerebral small vessel disease. However, radiologic studies investigating the associations between vasculature and basal ganglia are still lacking. Huge morphologic differences in LSA appearances among different individuals could be an important reason for the current limits of research on this topic, since currently available LSA imaging characteristics have poor sensitivity in being able to detect LSA abnormalities. In this study, the proposed method identified LSA vasculature and its supplying territories automatically. We showed a relationship between perforating arteries and the basal ganglia. The quantification of LSA vascular volume in subcortical areas could be used in both neurovascular studies and clinical diagnostic settings. LSA vascular volume reflects the density of perforating arteries and can be used to evaluate the impacts of lacunar infarcts, explore the causes of vascular dementia, and facilitate the etiologic studies of cerebral small vessel disease.

There are two major limitations to our study. First, large arteries were observed in the subcortical ROIs in 2 of 40 subjects, which interfered with the screening of LSA voxels and were thus removed from the analysis. These large vessels arose from the second MCA segments and crept along the surface of the brains without entering the parenchyma. Inclusion of these large arteries could be explained by misregistering subcortical ROIs from the templates to the individual spaces and should be improved upon in future studies. Second, when analyzing data from different subcortical areas, most vascular volume was found in the putamen, while very few voxels existed in other nuclei, especially the caudate nucleus. However, anatomic studies showed that LSAs arc around and course through the putamen and then travel superomedially through the superior part of the internal capsule and much of the caudate nucleus. The caudate nucleus is located superior to the putamen and other subcortical nuclei. The weakened in-flow of blood could have resulted in limited TOF-MRA sensitivity in the superior part of the scanning slab, and thus, miss small vessels in the caudate nucleus. However, the bias should not affect the results when comparing vascular volume in similar areas among different individuals.

\section{CONCLUSION}

In conclusion, we proposed an automatic processing pipeline for quantifying LSA vascular volume in subcortical regions. The results provided a general knowledge of the relationship between perforating arteries and basal ganglia. This method introduces a new dimension for interpreting LSA imaging findings, and could potentially assist in the radiologic evaluation of neurodegenerative diseases caused by small vascular lesions.

\section{DATA AVAILABILITY STATEMENT}

The raw data supporting the conclusions of this article will be made available by the authors, without undue reservation.

\section{ETHICS STATEMENT}

The studies involving human participants were reviewed and approved by The Institutional Review Board of Beijing MRI Center for Brain Research. The patients/participants provided their written informed consent to participate in this study.

\section{AUTHOR CONTRIBUTIONS}

NW and ZZ contributed to the conception and design of the study, analysis and interpretation of the data, and drafting of the manuscript. XZ and JA contributed to the acquisition of data. YZ contributed to the funding acquisition and supervision. All the authors approved the publication of the study.

\section{FUNDING}

This work was supported by the National Key R\&D Program of China (No. 2017YFC1307900, 2017YFC1307904), National Natural Science Foundation of China (82001804, 81961128030), Beijing Natural Science Foundation (7191003), Strategic Priority Research Program of Chinese Academy of Science (XDB32010300), and Capital's Funds for Health Improvement and Research (CFH2020-2-5115). 


\section{REFERENCES}

1. Marinković S, Gibo H, Milisavljević M, Cetković M. Anatomic and clinical correlations of the lenticulostriate arteries. Clin Anat. (2001) 14:1905. doi: 10.1002/ca.1032

2. Alexander L. The vascular supply of the strio-pallidum. Res Publ Assoc Res Nerv Ment Dis. (1942) 21:77-132.

3. Decavel P, Vuillier F, Moulin T. Lenticulostriate infarction. Front Neurol Neurosci. (2012) 30:115-9. doi: 10.1159/000333606

4. Jeans WD. The development and use of digital subtraction angiography. Brit J Radiol. (1990) 63:161-8. doi: 10.1259/0007-1285-63-747-161

5. Cho ZH, Kang CK, Han JY, Kim SH, Kim KN, Hong SM, et al. Observation of the lenticulostriate arteries in the human brain in vivo using 7.0T MR angiography. Stroke. (2008) 39:1604-6. doi: 10.1161/STROKEAHA.107.508002

6. Kang CK, Park CA, Lee H, Kim SH, Park CW, Kim YB, et al. Hypertension correlates with lenticulostriate arteries visualized by 7T magnetic resonance angiography. Hypertension. (2009) 54:1050-6. doi: 10.1161/HYPERTENSIONAHA.109.140350

7. Kang CK, Park CW, Han JY, Kim SH, Park CA, Kim KN, et al. Imaging and analysis of lenticulostriate arteries using 7.0-Tesla magnetic resonance angiography. Magn Reson Med. (2009) 61:136-44. doi: 10.1002/mrm.21786

8. Kong Q, Zhang Z, Yang Q, Fan Z, Wang B, An J, et al. 7T TOF-MRA shows modulated orifices of lenticulostriate arteries associated with atherosclerotic plaques in patients with lacunar infarcts. Eur J Radiol. (2019) 118:2716. doi: 10.1016/j.ejrad.2019.07.032

9. Seo SW, Kang CK, Kim SH, Yoon DS, Liao W, Worz S, et al. Measurements of lenticulostriate arteries using 7T MRI: new imaging markers for subcortical vascular dementia. J Neurol Sci. (2012) 322:2005. doi: 10.1016/j.jns.2012.05.032

10. Matsushige T, Chen B, Dammann P, Johst S, Quick HH, Ladd $\mathrm{ME}$, et al. Microanatomy of the subcallosal artery: an in-vivo $7 \mathrm{~T}$ magnetic resonance angiography study. Eur Radiol. (2016) 26:2908-14. doi: 10.1007/s00330-015-4117-1

11. Matsushige T, Kraemer M, Sato T, Berlit P, Forsting M, Ladd ME, et al. Visualization and classification of deeply seated collateral networks in moyamoya angiopathy with 7T MRI. AJNR Am J Neuroradiol. (2018) 39:124854. doi: 10.3174/ajnr.A5700

12. Ling C, Fang X, Kong Q, Sun Y, Wang B, Zhuo Y, et al. Lenticulostriate arteries and basal ganglia changes in cerebral autosomal dominant arteriopathy with subcortical infarcts and leukoencephalopathy, a high-field MRI study. Front Neurol. (2019) 10:870. doi: 10.3389/fneur.2019.00870

13. Kang CK, Park CA, Park CW, Lee YB, Cho ZH, Kim YB. Lenticulostriate arteries in chronic stroke patients visualised by $7 \mathrm{~T}$ magnetic resonance angiography. Int J Stroke. (2010) 5:374-80. doi: 10.1111/j.1747-4949.2010.00464.x

14. Yashiro S, Kameda H, Chida A, Todate Y, Hasegawa Y, Nagasawa K, et al. Evaluation of lenticulostriate arteries changes by $7 \mathrm{~T}$ magnetic resonance angiography in Type 2 diabetes. J Atheroscler Thromb. (2018) 25:106775. doi: $10.5551 /$ jat.43869

15. Ma SJ, Sarabi MS, Yan L, Shao X, Chen Y, Yang Q, et al. Characterization of lenticulostriate arteries with high resolution black-blood T1-weighted turbo spin echo with variable flip angles at 3 and 7 Tesla. Neuroimage. (2019) 199:184-93. doi: 10.1016/j.neuroimage.2019.05.065
16. Zhang Z, Fan Z, Kong Q, Xiao J, Wu F, An J, et al. Visualization of the lenticulostriate arteries at $3 \mathrm{~T}$ using black-blood T1-weighted intracranial vessel wall imaging: comparison with 7T TOF-MRA. Eur Radiol. (2019) 29:1452-9. doi: 10.1007/s00330-018-5701-y

17. Cho ZH, Lee YB, Kang CK, Yang JW, Jung IH, Park CA, et al. Microvascular imaging of asymptomatic MCA steno-occlusive patients using ultra-high-field 7T MRI. J Neurol. (2013) 260:144-50. doi: 10.1007/s00415-012-6604-5

18. Liem MK, van der Grond J, Versluis MJ, Haan J, Webb AG, Ferrari $\mathrm{MD}$, et al. Lenticulostriate arterial lumina are normal in cerebral autosomal-dominant arteriopathy with subcortical infarcts and leukoencephalopathy: a high-field in vivo MRI study. Stroke. (2010) 41:2812-6. doi: 10.1161/STROKEAHA.110.586883

19. Jenkinson M, Beckmann CF, Behrens TE, Woolrich MW, Smith SM. Fsl. Neuroimage. (2012) 62:782-90. doi: 10.1016/j.neuroimage.2011.09.015

20. Tustison NJ, Avants BB, Cook PA, Zheng Y, Egan A, Yushkevich PA, et al. N4ITK: improved N3 bias correction. IEEE Trans Med Imaging. (2010) 29:1310-20. doi: 10.1109/TMI.2010.2046908

21. Antiga L, Piccinelli M, Botti L, Ene-Iordache B, Remuzzi A, Steinman DA. An image-based modeling framework for patient-specific computational hemodynamics. Med Biol Eng Comput. (2008) 46:1097-112. doi: 10.1007/s11517-0080420-1

22. Kaplan HA, Ford DH. The Brain Vascular System. Amsterdam: Elsevier Publishing Company (1966).

23. Rosner SS, Rhoton AL, Ono M, Barry M. Microsurgical anatomy of the anterior perforating arteries. J Neurosurg. (1984) 61:468-85. doi: 10.3171/jns.1984.61.3.0468

24. Marinković SV, Kovačević MS, Marinković JM. Perforating branches of the middle cerebral artery: microsurgical anatomy of their extracerebral segments. J Neurosurg. (1985) 63:266-71. doi: 10.3171/jns.1985.63.2.0266

Conflict of Interest: XZ is an employee of Siemens Healthcare and JA is an employee of Siemens Shenzhen Magnetic Resonance Ltd. The Institute of Biophysics (Chinese Academy of Sciences) holds a research agreement with Siemens Healthcare.

The remaining authors declare that the research was conducted in the absence of any commercial or financial relationships that could be construed as a potential conflict of interest.

Publisher's Note: All claims expressed in this article are solely those of the authors and do not necessarily represent those of their affiliated organizations, or those of the publisher, the editors and the reviewers. Any product that may be evaluated in this article, or claim that may be made by its manufacturer, is not guaranteed or endorsed by the publisher.

Copyright (c) 2021 Wei, Zhang, An, Zhuo and Zhang. This is an open-access article distributed under the terms of the Creative Commons Attribution License (CC BY). The use, distribution or reproduction in other forums is permitted, provided the original author(s) and the copyright owner(s) are credited and that the original publication in this journal is cited, in accordance with accepted academic practice. No use, distribution or reproduction is permitted which does not comply with these terms. 